\title{
ZWINT positively regulates TBK1-mediated RLR antiviral signaling.
}

\author{
Sheng-Na Li, Shan-Shan Xu, Tian-Sheng He, Liang-Guo Xu* \\ Key Laboratory of Small Functional Organic Molecule, Ministry of Education, College of Life Science, Jiangxi Normal \\ University, Nanchang, Jiangxi, PR China
}

\begin{abstract}
TANK-Binding Kinase 1 (TBK1) is a serine/threonine kinase ubiquitously expressed in many tissues and plays an important role in the RLR antiviral signaling pathway. Upon viral infection, various virus components, including viral DNA, RNA are sensed by different pattern recognition receptors (PRRs). Among these PRRs, TLR3, RIG-I/MDA5, and cGAS recruit different adaptors including TRIF, VISA/ MAVS, and MITA/STING to activate TBK1. Activated TBK1 then promote the phosphorylation and homodimerization of IFN-regulatory factors 3 and 7 (IRF3/7), in turn promoting type-I Interferon (IFNI) production. In this study, we reported that ZW10 Interacting Kinetochore Protein (ZWINT) positively regulates RLR antiviral signaling by targeting RIG-I/VISA/TBK1. Ectopic expression of ZWINT potentiated Sendai virus-induced, as well as TBK1- or VISA/MAVS-mediated IFN- $\beta$ promoter activation by enhancing dimerization of IRF3. In contrast, knocking down of ZWINT expression with siRNA resulted in reduced IFN- $\beta$ promoter activation and IRF3 dimerization induced by Sendai virus, indicating that ZWINT positively regulates TBK1-mediated RLR antiviral signaling.
\end{abstract}

Keywords: ZWINT, RLR antiviral signaling, Interferon, IRF3, TBK1.

Accepted on May 30, 2018

\section{Introduction}

The viral nucleic acid or its intermediate metabolites, once bound to PRR, recruit the corresponding adapter protein, forming three major signaling pathways: RIG-I-VISA/MAVS, cGAS-STING, and TLR3-TRIF. The adaptor proteins VISA/ MAVS, STING and TRIF, utilize the protein kinase TBK1/ IKK $\varepsilon$ to promote IRF3 phosphorylation and induce type I interferons synthesis [1-4]. Therefore, TBK1 is a collection point for different antiviral signaling pathways, at the center of the antiviral signaling network.

RIG-I like receptor (RLR) is expressed in almost all cells and mainly recognizes viral dsRNA in the cytoplasm [5]. After RIG-I and MDA5 recognize the virus-stimulating signals, ATP-dependent conformational changes occur, forming dimmers and exposing the CARD structure, binding to the downstream common adapter protein VISA/MAVS via CARDCARD interactions [6-9]. It has been reported that in the RLR signaling pathway, VISA/MAVS activates TBK1/IKKe via TRAFs proteins, and the combined deletion of TRAF completely abolishes the MAVS-TBK1/IKKe interaction and subsequent TBK1/IKK $\varepsilon$ activation [8,10-12]. It was experimentally confirmed that MAVS recruits and activates TBK1/IKKe upon RNA virus infection through a preassociated TRAFs-TBK1/IKKe complex, resulting in activation of TBK1-IRF3 and type I IFN production [13].

TANK-Binding Kinase 1 (TBK1), belonging to IKK kinase family, is a very important kinase in RLR antiviral signaling. It contains distinct four domains, including a N-terminal Kinase Domain (KD), an ubiquitin-like domain (ULD) in the middle of TBK1, a Scaffold/Dimerization Domain (SDD) consists of Leucine Zipper (LZ), Helix-Loop-Helix (HLH) and the CTerminal Domain (CTD), which also known as Adaptor Binding (AB) motif [14,15]. TBK1 keeps an inactive state generally until the signaling is activated [14,15]. In the antiviral innate immune response, TBK1 and its upstream adapter protein, TRAFs, IRF3/7 constitute a functional complex, mutual activation between each other to form a complex network of antiviral signals [16]. Although it is well known that VISA/MAVS activates the transcription factor IRF3 via TBK1/IKKe after RNA virus infection, the mechanism by which TBK1/IKK $\varepsilon$ is activated remains unclear.

Here we identified ZW10 Interacting Kinetochore Protein (ZWINT) as a new TBK1 interaction protein through a yeast two-hybrid screening and co-immunoprecipitation. We discovered that ZWINT is a positive regulator of RLR antiviral signaling by associating RIG-I/VISA/TBK1complex. Our results revealed that ZWINT promoted Sendai virus induced TBK1 and VISA-mediated IRF3 dimerization and IFN- $\beta$ promoter activation. Knockdown of ZWINT inhibited Sendai virus induced IRF3 dimerization and the activation of IFN- $\beta$ promoter. In conclusion, ZWINT plays an important role in regulating the antiviral innate immune signaling pathway positively. 


\section{Materials and Methods}

\section{Construction of Plasmids}

Flag- or HA-tagged TBK1 and VISA expression plasmids for human, IFN- $\beta$ promoter luciferase reporter construct, IFNStimulated Response Element (ISRE) luciferase reporter plasmids were generously provided by Dr. Hong-Bing Shu (Wuhan University, Wuhan, China). Expression plasmids for human Flag- or HA-tagged ZWINT, HA-tagged actin were amplified by Polymerase Chain Reaction (PCR) and cloned into a pRK5'-Flag/HA vector. The three human ZWINT RNAi constructs were generated via the pSuper. Retro vector (Oligo Engine), following the manufacturer's protocols. The target sequences for the human ZWINT constructs were:

si\#1, CCAAGAAACAAATGGCCAT;

si\#2, CCTGCTGGAGATGTAAATT;

si\#3, GGAGATGTAAATTTGCCAT.

\section{Cell culture and stimuli}

$293 \mathrm{~T}$ cells were maintained at $37^{\circ} \mathrm{C}$ in Dulbecco modified Eagle's medium (DMEM; Gibco) supplemented with $10 \%$ heat-inactivated fetal bovine serum (FBS; Gibco) and 1\% penicillin/streptomycin in a $5 \% \mathrm{CO}_{2} / 95 \%$ air humidified incubator. Sendai virus was the gift from Dr. Hong-Bing Shu (Wuhan University, Wuhan, China).

\section{Dual-luciferase reporter assay}

$293 \mathrm{~T}$ cells $\left(\sim 2.5 \times 10^{5}\right)$ seeded in 24 -well plates were transfected with $100 \mathrm{ng}$ IFN- $\beta$ promoter luciferase reporter plasmid or ISRE luciferase reporter construct(firefly luciferase), $50 \mathrm{ng}$ pRL-TK (renilla luciferase plasmid) and indicated amount of other expression plasmids or empty vector through the calcium phosphate method. Twelve hours after transfection, we treated cells with $(+)$ or without (-) Sendai virus for indicated time periods in some experiments, and then cells were harvested and lysed by $1 \mathrm{X}$ passive lysis buffer. Whereafter, we used a dual-luciferase reporter assay system (Promega) with a GloMax ${ }^{\mathrm{TM}}$ 20/20 Luminometer (Promega) to assess luciferase activity according to the instructions provided by the manufacturer and the ratio of firefly luciferase activity to Renilla luciferase activity for normalization. We repeated the experiments for three times, and the data was the average value.

\section{Immunoprecipitation, immunoblotting and antibodies}

$293 \mathrm{~T}$ cells $\left(\sim 6 \times 10^{6}\right)$ were cultured in $100 \mathrm{~mm}$ dishes and transfected with indicated plasmids. Twelve-hours after transfection, cells were infected with $(+)$ or without(-) Sendai virus for the indicated time, and then the cells were collected and lysed with lysis buffer, which consisted of $150 \mathrm{mM} \mathrm{NaCl}$, $20 \mathrm{mM}$ Tris- $\mathrm{HCl}$ (pH 7.5), 1 mM EDTA, 1\% Triton X-100, 1 mM PMSF, $1 \mathrm{M} \mathrm{NaF}, 1 \mathrm{M} \mathrm{Na}_{3} \mathrm{VO}_{4}, 10 \mu \mathrm{g} / \mathrm{ml}$ aprotinin and 10 $\mu \mathrm{g} / \mathrm{ml}$ leupeptin. And then $350 \mu \mathrm{l}$ lysate was incubated at $4^{\circ} \mathrm{C}$ with indicated amount of Flag antibody or control antibody and $\sim 30 \mu \mathrm{l}$ protein G/A-Sepharose beads in 20\% ethanol (GE Healthcare, Piscataway, NJ) overnight for each immunoprecipitation. The sepharose beads were washed once with lysis buffer containing $1 \mathrm{M} \mathrm{NaCl}$, the precipitates were separated by SDS-PAGE, and Western blot analysis was performed by standard immunoblot procedures. Further, the sample proteins were transferred to Hybond nitrocellulose membrane (Pall Corporation, USA). The immunoblot was performed by using the indicated primary antibodies including the mouse anti-Flag (1/4000), anti-HA (1/1000) monoclonal antibody (Sigma), the rabbit Antibodies (Abs) against IRF3 (1/1000,sc-9082, Santa Cruz).

\section{Real-time fluorescent quantitative PCR}

Total RNA was isolated from cultured cells using RNAextraction kit (Promega) according to manufacturer's protocols and cDNA was reverse transcribed using a reverse transcription kit (Promega) for real-time fluorescent quantitative PCR analysis with a SYBR quantitative PCR kit (Promega). Data are presented as expression levels compared with $\beta$-actin. All primers for the real-time PCR used are listed as the following:

hZWINT forward, GGAAGCCCAGAGGAAACG, hZWINT reverse, CAGAAACCTCCGCCAGATG;

hIFN- $\beta$ forward, TGGGAGGATTCTGCATTACC, hIFN- $\beta$ reverse, CAGCATCTGCTGGTTGAAGA;

$\mathrm{SeV}$ forward, AAACGCATCACGTCTCTTCC, SeV reverse, TTCTCAGCTCTGCTTAGGGG;

$\beta$-actin forward, GTCGTCGACAACGGCTCCGGCATG,

$\beta$-actin reverse, ATTGTAGAAGGTGTGGTGCCAGAT.

\section{Results}

\section{Identification of ZWINT as a partner of RIG-I/VISA/ TBK1 antiviral signalosome}

TANK-Binding Kinase 1 (TBK1) plays a key role in RIG-IVISA and other antiviral innate pathways. Upon viral infection, activated RIG-I translocate to the mitochondria outer membrane, where it binds to VISA and activates TBK1 to regulate phosphorylation of IRF3, resulting in the induction of type I interferons after virus infection $[17,18]$. In this process, TBK1 plays a key regulatory role, but TBK1 regulatory mechanism remains to be further studied. To further investigate the mechanism of TBK1 function in host antiviral immune responses, we screened ZWINT by yeast two-hybrid system as one of candidate TBK1 associated proteins. To evaluate whether ZWINT interacts with TBK1 or other RLR signaling partner in mammalian cells, we co-transfected HA-tagged ZWINT together with Flag-tagged TBK1, VISA, and RIG-I, respectively, and performed coimmunoprecipitation and immunoblot assays in $293 \mathrm{~T}$ cells, the results showed that ZWINT interacts with TBK1, VISA/MAVS and RIG-I (Figure 
1). This data suggests that ZWINT may associate with TBK1 and RIG-I-mediated RLR antiviral signaling.

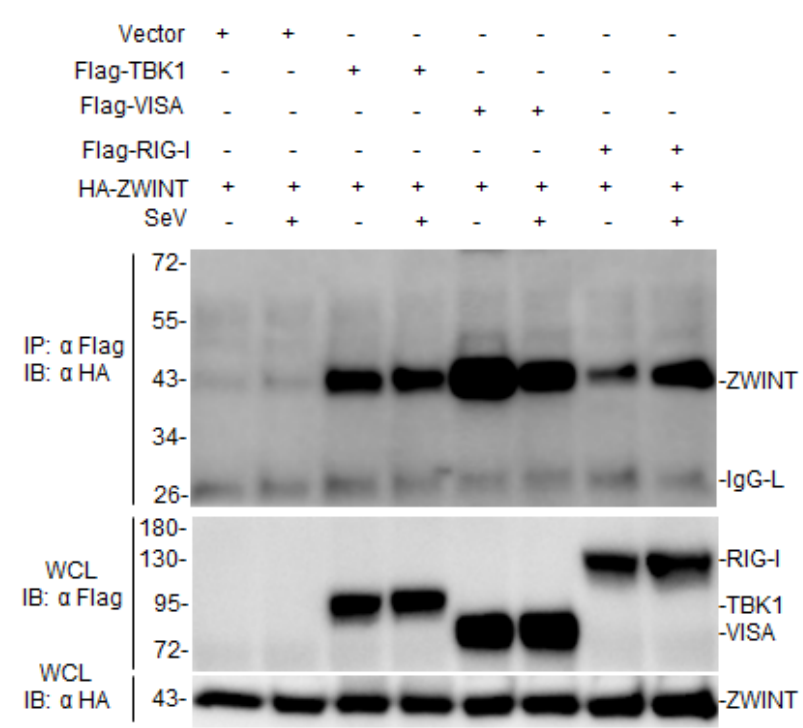

Figure 1. ZWINT is a partner of RIG-I/VISA/TBK1 antiviral signalosome. $293 \mathrm{~T}$ cells $\left(\sim 1 \times 10^{7}\right)$ seeded in $100 \mathrm{~mm}$ dishes were transfected with HA-tagged ZWINT (10 $\mu \mathrm{g}$ each) and HA-tagged actin ( $1 \mu \mathrm{g}$, as the internal control), together with empty vector, or Flag-tagged-VISA (10 $\mu \mathrm{g})$, or Flag-tagged-TBK1, or Flag-tagged RIG-I (10 $\mu \mathrm{g}$ each). Twelve hours after transfection, cells were infected with or without Sendai virus for $12 \mathrm{~h}$, then cells were collected and lysed, and the lysate were analysed by a coimmunoprecipitate with anti-Flag and immunoblot analysis with anti-HA (upper panels). Expression of other proteins were analysed by immunoblotting with anti-Flag, anti-HA and anti-actin (lower panels).

\section{ZWINT not only enhances Sendai virus-induced but also enhances TBK1 and VISA-mediated antiviral signals}

To test whether ZWINT is involved in TBK1 and RLRmediated antiviral signaling, we performed dual-luciferase reporter assays to determinate whether ZWINT could activate or inhibit IFN- $\beta$ promoter or ISRE, as shown in Figure 2A, over-expression of ZWINT didn't have the ability to activate or inhibit IFN- $\beta$ promoter and ISRE, but potentiated Sendai virusinduced activation of IFN- $\beta$ promoter and ISRE. Real-time fluorescent quantitative PCR data also showed that overexpression of ZWINT increased the production of IFN- $\beta$ induced by Sendai virus (Figure 2B). Furthermore, ZWINT over-expression also potentiated TBK1 and VISA/MAVSmediated activation of the IFN- $\beta$ promoter and ISRE in a dosedependent manner in reporter assays (Figures 2C and 2D).

Since the transcriptional factor IRF3 is a pivotal molecule to facilitate the induction of IFN- $\beta$ in RLR pathway [19]. Consistently, over-expression of ZWINT markedly increases the abundance of Sendai virus-triggered IRF3 dimerization (Figure 2E). Furthermore, Overexpression of TBK1 and VISAmediated IRF3 dimerization was enhanced by ZWINT in a dose-dependent manner (Figures $2 \mathrm{~F}$ and $2 \mathrm{G}$ ).

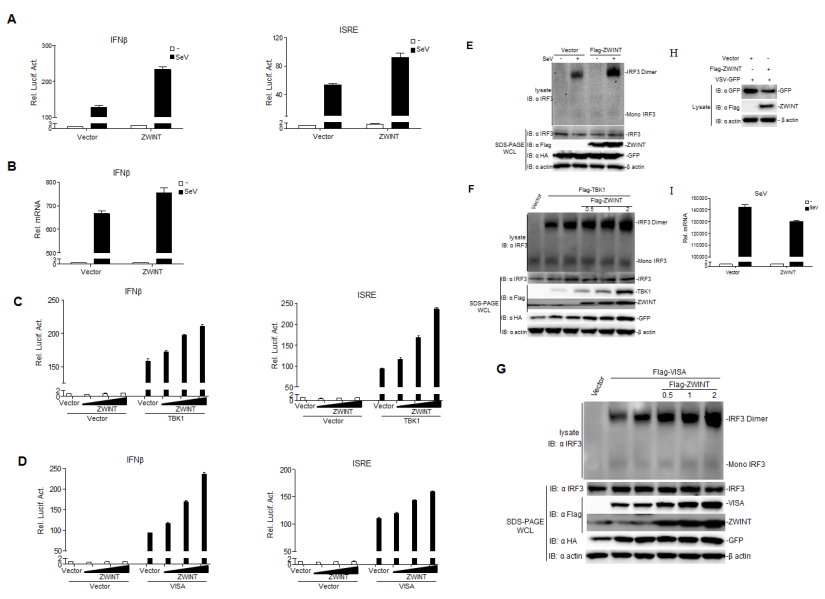

Figure 2. ZWINT potentiates Sendai virus-induced, RLR-mediated antiviral signaling. (A): ZWINT potentiates Sendai virus-induced IFN- $\beta$ promoter and ISRE activation in reporter assays. 293 T cells $(2$ $\times 10^{5}$ ) seeded in 24 well plates were transfected with an IFN- $\beta$ promoter luciferase plasmid $(0.1 \mu \mathrm{g})$ or ISRE luciferase plasmids $(0.1$ $\mu \mathrm{g})$ and the indicated mammalian expression plasmids $(0.5 \mu \mathrm{g}$ each). 12 h after transfection, cells were treated with (+) or without Sendai virus for $12 \mathrm{~h}$, then cells were harvested and lysed for luciferase assay. (B): ZWINT potentiates Sendai virus-induced IFN- $\beta$ production. $293 \mathrm{~T}$ cells $\left(1 \times 10^{6}\right)$ seeded in 6 well dishes were transfected with ZWINT plasmid (1.0 $\mu \mathrm{g}$ each) or control plasmid. 12 $h$ after transfection, cells were treated with (+) or without Sendai virus for $12 \mathrm{~h}$, then cells were harvested, the mRNA levels of IFN- $\beta$ were analysed by a qPCR assay. (C): ZWINT enhances TBK1mediated IFN- $\beta$ promoter and ISRE activation in reporter assays. $293 T$ cells $\left(\sim 2 \times 10^{5}\right)$ seeded in 24 well plates were transfected with an IFN- $\beta$ promoter luciferase plasmid $(0.1 \mu \mathrm{g})$ or ISRE luciferase plasmids $(0.1 \mu \mathrm{g})$ and the mammalian TBK1 expression plasmids or control plasmids, together with an increased amount of ZWINT expression plasmid (0.02, 0.05, $0.1 \mu \mathrm{g}$ each). $18 \mathrm{~h}$ after transfection, cells were harvested and lysed for luciferase assay. (D): ZWINT enhances VISA-mediated IFN- $\beta$ promoter and ISRE activation in reporter assays. $293 T$ cells $\left(2 \times 10^{5}\right)$ seeded in 24 well plates were transfected with an IFN- $\beta$ promoter luciferase plasmid $(0.1 \mu \mathrm{g})$ or ISRE luciferase plasmids $(0.1 \mu \mathrm{g})$ and the mammalian VISA expression plasmids or control plasmids, together with increased amount of ZWINT expression plasmid (0.02, 0.05, $0.1 \mu \mathrm{g}$ each). $18 \mathrm{~h}$ after transfection, cells were harvested and lysed for luciferase assay. (E): ZWINT reinforces Sendai virus-induced IRF3 activation. $293 T$ cells $\left(1 \times 10^{6}\right)$ seeded in 6 well dishes were transfected with ZWINT plasmid (1.0 $\mu \mathrm{g}$ each) or control plasmid. $12 \mathrm{~h}$ after transfection, cells were treated with (+) or without Sendai virus for $12 \mathrm{~h}$, cells were harvested and IRF3 dimerization was detected by running native PAGE gel. (F and G): ZWINT reinforces TBK1 and VISAmediated IRF3 activation in a dose-dependent manner. 293 cells (1 $\times 10^{6}$ ) seeded in 6 well dishes were transfected with TBK1 or VISA and control plasmids, together with different dosage of ZWINT plasmid $(0.5,1.0,2.0 \mu \mathrm{g}$ each). $18 \mathrm{~h}$ after transfection, cells were harvested and IRF3 dimerization was detected by running native PAGE gel. (H): ZWINT inhibits the expression of vesicular stomatitis virus. $293 T$ cells $\left(1 \times 10^{6}\right)$ seeded in 6 well dishes were transfected with Flag-ZWINT plasmid (5.0 $\mu \mathrm{g}$ each) and control plasmid, $12 \mathrm{~h}$ after transfection, cells were treated with (+) VSV-GFP for $12 \mathrm{~h}$, then cells were harvested and lysed for immunoblotting with antibodies against GFP, Flag and $\beta$-actin respectively. (I): ZWINT inhibits the transcription of SeV. $293 \mathrm{~T}$ cells $\left(1 \times 10^{6}\right)$ seeded in 6 well dishes were transfected with ZWINT plasmid $(3.0 \mu \mathrm{g}$ each) and control plasmid, $12 \mathrm{~h}$ after transfection, cells were treated with (+) or without Sendai virus for $12 \mathrm{~h}$, then cells were harvested, the $\mathrm{mRNA}$ levels of SeV were analysed by a qPCR assay. 


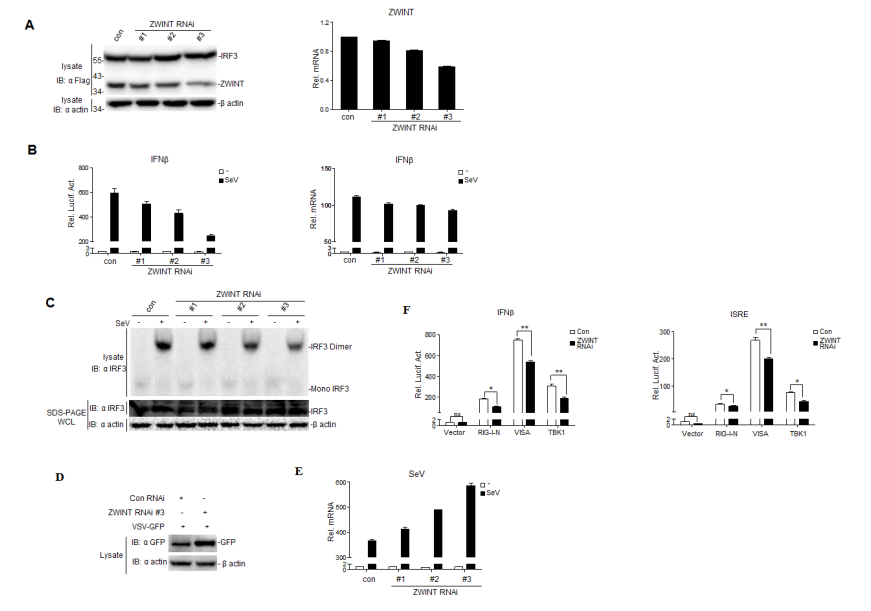

Figure 3. Knockdown of ZWINT inhibits RLR antiviral signaling. (A): Effects of ZWINT-RNAi plasmids on expression of transfected ZWINT (left) or endogenous ZWINT (right). $293 T$ cells $\left(1 \times 10^{6}\right)$ seeded in 6 well dishes were transfected with expression plasmids for FlagZWINT and IRF3 (2 $\mu \mathrm{g}$ each), and the indicated ZWINT RNAi plasmids or control plasmids (2 $\mu \mathrm{g}$ each), thirty-six hours after transfection, cell lysates were analysed by immunoblot with antibodies against Flag and $\beta$-actin respectively (left). Effects of ZWINT-RNAi on endogenous expression of ZWINT at mRNA level. $293 T$ cells $\left(1 \times 10^{6}\right)$ seeded in 6 well dishes were transfected control RNAi or indicated ZWINT RNAi (2 $\mu \mathrm{g}$ each) for $36 \mathrm{~h}$ before qPCR assay analysis for measuring the endogenous ZWINT expression at mRNA level (right). (B): Effects of ZWINT RNAi on Sendai virusinduced IFN- $\beta$ promoter activation and IFN- $\beta$ production. (Left) The 293 T cells $\left(2 \times 10^{5}\right)$ were transfected with the indicated control RNAi or ZWINT RNAi plasmids (1 $\mu \mathrm{g}$ each) and IFN- $\beta$ promoter luciferase reporter plasmids. Twenty-four hours after transfection, cells were infected with or without Sendai virus for $12 \mathrm{~h}$ before reporter assays were performed. (Right) The $293 \mathrm{~T}$ cells $\left(4 \times 10^{5}\right)$ were transfected with the indicated control RNAi or ZWINT RNAi plasmids $(2 \mu \mathrm{g}$ each). Twenty-four hours after transfection, cells were infected with or without Sendai virus for $12 \mathrm{~h}$ before $q P C R$ analysis for IFN- $\beta$ production at mRNA level was performed. (C): Effects of ZWINT RNAi on Sendai virus-induced IRF3 dimerization. The 293 T cells (1 $\left.\times 10^{6}\right)$ seeded in 6 well dishes were transfected with the indicated control RNAi or ZWINT RNAi plasmids (2 $\mu \mathrm{g}$ each). Twenty-four hours after transfection, cells were infected with or without Sendai virus for $12 \mathrm{~h}$ before native PAGE gel analysis for IRF3 dimerization was performed. (D): Effects of ZWINT RNAi on the expression of vesicular stomatitis virus. $293 T$ cells $\left(1 \times 10^{6}\right)$ seeded in 6 well dishes were transfected with ZWINT RNAi \#3 plasmids and control RNAi plasmids (5 $\mu \mathrm{g}$ each), twelve hours after transfection, cells were treated with (+) VSV-GFP for $12 \mathrm{~h}$, then cell lysates were analysed by immunoblot with antibodies against GFP and $\beta$-actin respectively. (E): Effects of ZWINT RNAi on the transcription of Sendai virus. $293 T$ cells $\left(1 \times 10^{6}\right)$ seeded in 6 well dishes were transfected with control RNAi plasmid and indicated ZWINT RNAi plasmid (3.0 $\mu \mathrm{g}$ each). Twelve hours after transfection, cells were infected with (+) or without Sendai virus for $12 \mathrm{~h}$ before qPCR analysis for $\mathrm{SeV}$ production at mRNA level was performed. $(F)$ : Effects of ZWINT RNAi on TBKI/VISA/RIG-I-mediated IFN- $\beta$ promoter and ISRE activation. $293 T$ cells $\left(\sim 2 \times 10^{5}\right)$ seeded in 24 well plates were transfected with an IFN- $\beta$ promoter luciferase plasmid $(0.1 \mu \mathrm{g})$ or ISRE luciferase plasmids $(0.1 \mu \mathrm{g})$ and the control RNAi and ZWINT RNAi \#3 plasmids (2 $\mu \mathrm{g}$ each), together with the indicated RLR signaling expression plasmids or control plasmids (1 $\mu \mathrm{g}$ each). Eighteen hours after transfection, cells were harvested and lysed for luciferase assay.
Further, over-expression of ZWINT inhibited the expression of Vesicular Stomatitis Virus (VSV) (Figure 2H) and inhibited the replication of $\mathrm{SeV}$ (Figure 2I) observably by the results of immunoblot and real-time fluorescent quantitative PCR respectively. Taken together, these data suggest that ZWINT acts as a positive regulator of RLR antiviral signaling pathway.

\section{Knockdown of $Z$ WINT inhibits IFN- $\beta$ induction and antiviral responses}

To address the role of ZWINT on RLR antiviral signaling, we constructed three RNAi plasmids, pSuper-ZWINT-RNAi\#1-3, by targeting three different ZWINT specific sequence, respectively, to knockdown ZWINT expression. We cotransfected the Flag-ZWINT and IRF3 plasmids together with a siRNA control or ZWINT-specific siRNAs into 293T cells, immunoblot analysis indicated that all these three RNAi could inhibit the expression of overexpressed ZWINT (Figure 3A left). Meanwhile, we also transfected all the ZWINT RNAi plasmids into $293 \mathrm{~T}$ cells to verify that all three RNAi has the ability to knockdown ZWINT expression at the mRNA level by using the real-time fluorescent quantitative PCR (Figure 3A right). Both immunoblotting and $\mathrm{qPCR}$ results showed that ZWINT RNAi\#3 was the best one to knockdown ZWINT expression.

We then performed a reporter assays to address whether knockdown of the endogenous ZWINT expression effects Sendai virus-induced or TBK1 and VISA/MAVS-mediated IFN- $\beta$ promoter activation. As shown in Figure 3B left, knockdown of the expression of ZWINT repressed Sendai virus-induced IFN- $\beta$ promoter activation, and real-time fluorescent quantitative PCR results showed that knockdown of ZWINT expression reduced Sendai virus-induced IFN- $\beta$ expression at the level of mRNA (Figure 3B right).

Moreover, we found that knockdown of ZWINT remarkably reduced the endogenous IRF3 dimerization induced by Sendai virus (Figure 3C). Consistently, immunoblot (Figure 3D) and real-time fluorescent quantitative PCR (Figure 3E) showed an increased expression quantity of VSV and $\mathrm{SeV}$ respectively by knockdown of the expression of ZWINT. Our results demonstrate that knockdown of ZWINT reduced RLR antiviral responses by weakening the activation of IFN- $\beta$ promoter and IRF3.

Besides, dual-luciferase reporter assays showed that knockdown of the expression of ZWINT inhibited TBK1, VISA and RIG-I-mediated IFN- $\beta$ promoter activity (Figure $3 F$ ), indicating that ZWINT can target TBK1 to regulate RLR antiviral signals, as well as VISA and RIG-I.

\section{ZWINT enhances polyubiquitination of TBK1}

Because ZWINT enhances TBK1-mediated antiviral signaling, we next examined whether ZWINT could promote K63-linked polyubiquitination of TBK1. To determine whether ZWINT regulates ubiquitination of TBK1, we transfected 293T cells with Flag-tagged-TBK1 and HA-tagged ubiquitin, or K63 only ubiquitin, or K48 only ubiquitin plasmids, together with myc- 
tagged-ZWINT or empty vector as a control, $12 \mathrm{~h}$ after transfection, the cells were infected with or without Sendai virus, then we harvested the cells and performed a coimmunoprecipitation assays using anti-Flag. The results showed that ZWINT markedly augmented ubiquitination of TBK1, and this ubiquitination increased more following Sendai virus infection. Further experiments showed that ZWINT mainly promoted K63-linked polyubiquitination of TBK1 (Figure 4). The results suggested that ZWINT positively regulates RLR-mediated antiviral signaling by promoting K63linked polyubiquitination of TBK1 when RLR-VISA antiviral signaling is activated.

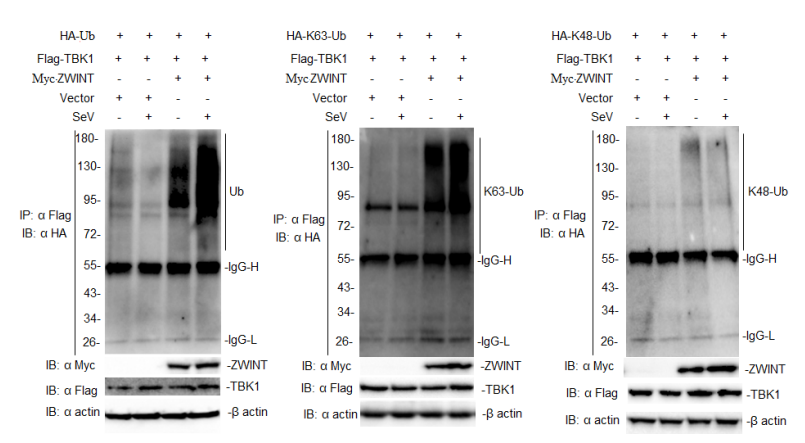

Figure 4. ZWINT promotes K63-linked polyubiquitination of TBK1. The $293 T$ cells $\left(1 \times 10^{7}\right)$ were transfected with indicated plasmids. Sixteen hours after transfection, cells were treated with or without Sendai virus for $12 \mathrm{~h}$, then cells were harvested, and ubiquitination assays were performed, respectively for wild type-linked (left), K63linked (middle) or K48-linked (right) polyubiquitination.

\section{Discussion}

In this study, we discovered a novel TBK1 and RIG-I/VISA associated partner through a yeast two-hybrid and coimmunoprecipitation. We demonstrated that over-expression of ZWINT potentiates IRF3 phosphorylation and IFN- $\beta$ promoter activation induced by Sendai virus or mediated by TBK1 and VISA/MAVS (Figure 2), suggesting that ZWINT is positively involved in RLR antiviral signaling by targeting TBK1/VISA/ RIG-I. Our study first uncovered ZWINT has a function in antiviral immune signaling. It has not been reported so far that ZWINT is involved in antiviral immune response.

ZWINT, an essential Zeste White 10 (ZW10) partner, is required for kinetochore localization of Zeste White 10 (ZW10) by bridging the specific association between the kinetochore and ZW10 [20,21]. Previous studies showed that ZWINT is involved in the processes of mitosis and meiosis, by working as a part of the kinetochore scaffold to recruit ZW10 to the kinetochore, making accurate chromosome segregation and genetic stability in mitosis and meiosis [22-24]. ZWINT also adjusts neurotransmitter release and is regulated in peripheral nerve injury-induced neuropathic pain $[25,26]$.

ZWINT is a substrate of Aurora B kinase [24,27], which plays an important role in mitosis [28-31]. It is reported that abolishment of Aurora B made an enhancive specific infectivity of $\mathrm{HCV}$ [32]. In addition, inhibition of Aurora kinase mediates HTLV-1-infected T lymphocytes growth and survival negatively [33]. All these suggested that Aurora B involves in antiviral immune signaling. Here we discovered that ZWINT, a mitosis regulator, is involved in RLR antiviral signaling by enhancing Sendai virus-induced or TBK1 and VISA-mediated activation of IRF3 and IFN- $\beta$ promoter by targeting TBK1/VISA/RIG-I. We hypothesize there is a tight relationship between antiviral immune signaling and mitosis but with a blurry mechanism, more essential mechanism how ZWINT involves in TBK1/VISA/RIG-I-mediated RLR antiviral immune signaling is still infusive enigma to be further studied.

\section{Acknowledgement}

We are grateful to Dr. Hong-Bing Shu (Medical Research Institute, Wuhan University) for his helping for providing plasmids and other reagents assistance. This work was financially supported by National Natural Science Foundation of China (31370876, 31570876), Natural Science Foundation of Jiangxi Province (20143ACB20004, 20161BAB204177), and the Open Project Program of Key Laboratory of Functional Small organic molecule, Ministry of Education, Jiangxi Normal University (No. KLFS-KF-201407). The authors declare no competing financial interests.

\section{References}

1. Liu S, Cai X, Wu J, Cong Q, Chen X, Li T, Du F, Ren J, Wu Y T, Grishin N V, Chen ZJ. Phosphorylation of innate immune adaptor proteins MAVS, STING, and TRIF induces IRF3 activation. Science 2015; 347: 2630.

2. Yoneyama M, Fujita T. RNA recognition and signal transduction by RIG-I-like receptors. Immunol Rev 2009; 227: 54-65.

3. Ishikawa H, Barber GN. STING is an endoplasmic reticulum adaptor that facilitates innate immune signalling. Nature 2008; 455: 674-678.

4. Oshiumi H, Matsumoto M, Funami K, Akazawa T, Seya T.TICAM-1, an adaptor molecule that participates in Tolllike receptor 3-mediated interferon-beta induction. Nat Immunol 2003; 4: 161-167.

5. Akira S, Uematsu S, Takeuchi O. Pathogen recognition and innate immunity. Cell 2006; 124: 783-801.

6. Chiang JJ, Davis ME, Gack MU. Regulation of RIG-I-like receptor signaling by host and viral proteins. Cytokine Growth Factor Rev 2014; 25: 491-505.

7. Kowalinski E, Lunardi T, McCarthy AA, Louber J, Brunel J, Grigorov B, Gerlier D, Cusack S. Structural basis for the activation of innate immune pattern-recognition receptor RIG-I by viral RNA. Cell 2011; 147: 423-435.

8. Xu LG, Wang YY, Han KJ, Li LY, Zhai Z, Shu HB. VISA is an adapter protein required for virus-triggered IFN-beta signaling. Mol Cell 2005; 19: 727-740.

9. Wu B, Hur S. How RIG-I like receptors activate MAVS. Curr Opin Virol 2015; 12: 91-98.

10. Fang R, Jiang Q, Zhou X, Wang C, Guan Y, Tao J, Xi J, Feng JM, Jiang Z. MAVS activates TBK1 and IKKepsilon 
through TRAFs in NEMO dependent and independent manner. PLoS Pathog 2017; 13: 1006720.

11. Tang ED, Wang CY. TRAF5 is a downstream target of MAVS in antiviral innate immune signaling. PLoS One 2010; 5: 9172.

12. Saha SK, Pietras EM, He JQ, Kang JR, Liu SY, Oganesyan G, Shahangian A, Zarnegar B, Shiba TL, Wang Y, Cheng G. Regulation of antiviral responses by a direct and specific interaction between TRAF3 and Cardif. Embo J 2006; 25: 3257-3263.

13. Bakshi S, Taylor J, Strickson S, McCartney T, Cohen P. Identification of TBK1 complexes required for the phosphorylation of IRF3 and the production of interferon beta. Biochem J 2017; 474: 1163-1174.

14. Helgason E, Phung QT, Dueber EC. Recent insights into the complexity of Tank-binding kinase 1 signaling networks: the emerging role of cellular localization in the activation and substrate specificity of TBK1. FEBS Lett 2013; 587: 1230-1237.

15. Tu D, Zhu Z, Zhou AY, Yun CH, Lee KE, Toms AV, Li Y, Dunn GP, Chan E, Thai T, Yang S, Ficarro SB, Marto JA, Jeon H, Hahn WC, Barbie DA, Eck MJ. Structure and ubiquitination-dependent activation of TANK-binding kinase 1. Cell Rep 2013; 3: 747-758.

16. Chau TL, Gioia R, Gatot JS, Patrascu F, Carpentier I, Chapelle JP, ONeill L, Beyaert R, Piette J, Chariot A. Are the IKKs and IKK-related kinases TBK1 and IKK-epsilon similarly activated? Trends Biochem Sci 2008; 33: 171-180.

17. McWhirter SM, Fitzgerald KA, Rosains J, Rowe DC, Golenbock DT, Maniatis T. IFN-regulatory factor 3dependent gene expression is defective in Tbk1-deficient mouse embryonic fibroblasts. Proc Natl Acad Sci USA 2004; 101: 233-238.

18. Fitzgerald KA, McWhirter SM, Faia KL, Rowe DC, Latz E, Golenbock DT, Coyle AJ, Liao SM, Maniatis T. IKKepsilon and TBK1 are essential components of the IRF3 signaling pathway. Nat Immunol 2003; 4: 491-496.

19. Khan KA, Do F, Marineau A, Doyon P, Clement JF, Woodgett JR, Doble BW, Servant MJ. Fine-tuning of the RIG-I-like receptor/interferon regulatory factor 3dependent antiviral innate immune response by the glycogen synthase kinase 3/beta-catenin pathway. Mol Cell Biol 2015; 35: 3029-3043.

20. Starr DA, Saffery R, Li Z, Simpson AE, Choo KH, Yen TJ, Goldberg ML. HZwint-1, a novel human kinetochore component that interacts with HZW10. J Cell Sci 2000; 113: 1939-1950.

21. Wang H, Hu X, Ding X, Dou Z, Yang Z, Shaw AW, Teng M, Cleveland DW, Goldberg ML, Niu L, Yao X. Human Zwint-1 specifies localization of Zeste White 10 to kinetochores and is essential for mitotic checkpoint signaling. J Biol Chem 2004; 279: 54590-54598.

22. Famulski JK, Vos L, Sun X, Chan G. Stable hZW10 kinetochore residency, mediated by hZwint-1 interaction, is essential for the mitotic checkpoint. J Cell Biol 2008; 180: 507-520.

23. Vos Larissa J, Famulski Jakub K, Chan Gordon KT. hZwint-1 bridges the inner and outer kinetochore: identification of the kinetochore localization domain and the hZw10-interaction domain. Biochem J 2011; 436: 157-168.

24. Woo Seo D, Yeop You S, Chung WJ, Cho DH, Kim JS, $\mathrm{Su} \mathrm{Oh} \mathrm{J}$. Zwint-1 is required for spindle assembly checkpoint function and kinetochore-microtubule attachment during oocyte meiosis. Sci Rep 2015; 5: 15431.

25. Han M, Xiao X, Yang Y, Huang RY, Cao H, Zhao ZQ, Zhang YQ. SIP30 is required for neuropathic pain-evoked aversion in rats. J Neurosci 2014; 34: 346-355.

26. Peng G, Han M, Du Y, Lin A, Yu L, Zhang Y, Jing N. SIP30 is regulated by ERK in peripheral nerve injuryinduced neuropathic pain. J Biol Chem 2009; 284: 30138-30147.

27. Kasuboski JM, Bader JR, Vaughan PS, Tauhata SB, Winding M, Morrissey MA, Joyce MV, Boggess W, Vos L, Chan GK, Hinchcliffe EH, Vaughan KT. Zwint-1 is a novel Aurora B substrate required for the assembly of a dynein-binding platform on kinetochores. Mol Biol Cell 2011; 22: 3318-3330.

28. Famulski JK, Chan GK. Aurora B kinase-dependent recruitment of $\mathrm{hZW10}$ and hROD to tensionless kinetochores. Curr Biol 2007; 17: 2143-2149.

29. Hsu JY, Sun ZW, Li X, Reuben M, Tatchell K, Bishop DK, Grushcow JM, Brame CJ, Caldwell JA, Hunt DF, Lin R, Smith MM, Allis CD. Mitotic phosphorylation of histone $\mathrm{H} 3$ is governed by Ipl1/aurora kinase and Glc7/PP1 phosphatase in budding yeast and nematodes. Cell 2000; 102: 279-291.

30. Welburn JPI, Vleugel M, Liu D, Yates Iii JR, Lampson MA, Fukagawa T, Cheeseman IM. Aurora B phosphorylates spatially distinct targets to differentially regulate the kinetochore-microtubule interface. Mol Cell 2010; 38: 383-392.

31. Carmena M, Ruchaud S, Earnshaw WC. Making the Auroras glow: regulation of Aurora A and B kinase function by interacting proteins. Curr Opin Cell Biol 2009; 21: 796-805.

32. Madejon A, Sheldon J, Francisco-Recuero I, Perales C, Dominguez-Beato M, Lasa M, Sanchez-Perez I, Muntane J, Domingo E, Garcia-Samaniego J, Sanchez-Pacheco A. Hepatitis C virus-mediated Aurora B kinase inhibition modulates inflammatory pathway and viral infectivity. J Hepatol 2015; 63: 312-319.

33. Tomita M, Tanaka Y, Mori N. Aurora kinase inhibitor AZD1152 negatively affects the growth and survival of HTLV-1-infected $\mathrm{T}$ lymphocytes in vitro. Int $\mathrm{J}$ Cancer 2010; 127: 1584-1594. 
ZWINT positively regulates TBK1-mediated RLR antiviral signaling

\section{*Correspondence to}

Liang-Guo Xu

Key Laboratory of Small Functional Organic Molecule

Ministry of Education

College of Life Science

Jiangxi Normal University

PR China 